\title{
Rerendering Landscape Photographs
}

\author{
Pu Wang \\ Diana Bicazan \\ Imperial College London* \\ Abhijeet Ghosh
}

\begin{abstract}
We present a practical approach for realistic rerendering of landscape photographs. We extract a view dependent depth map from single input landscape images by examining global and local pixel color distributions and demonstrate applications of depth dependent rendering such as novel viewpoints, digital refocusing and dehazing. We also present a simple approach to relight the input landscape photograph under novel sky illumination. Here, we assume diffuse reflectance and relight landscapes by estimating the irradiance due the sky in the input photograph. Finally, we also take into account specular reflections on water surfaces which are common in landscape photography and demonstrate a semiautomatic process for relighting scenes with still water.
\end{abstract}

\section{Keywords}

Depth estimation, relighting.

\section{INTRODUCTION}

The field of computer graphics has constantly been striving towards increased realism in rendered imagery. Realistic image synthesis has major applications in the entertainment industry in visual effects for movies and games, virtual reality, architectural visualization, and emergent applications such as product advertising. The realism of rendered imagery is however often dependent on the quality of input scene description in terms of scene geometry and reflectance. Advances in digital photography over the last decade has thus given rise to image based techniques for modeling and rendering for realistic computer graphics. In this work, we propose an image based approach for modeling and rendering of a specific type of scene - natural landscapes. We focus on landscape images in this work because they are ubiquitous, e.g., in personal photo collections or the web. They also find many applications in computer generated imagery, e.g., forming scenic backgrounds in visual effects shots for movies and advertisements. Landscapes are aesthetically beautiful and interesting, due to which they have always attracted photographers. This is why striking imagery of natural

*e-mail:ghosh@imperial.ac.uk

Permission to make digital or hard copies of all or part of this work for personal or classroom use is granted without fee provided that copies are not made or distributed for profit or commercial advantage and that copies bear this notice and the full citation on the first page. Copyrights for components of this work owned by others than ACM must be honored. Abstracting with credit is permitted. To copy otherwise, or republish, to post on servers or to redistribute to lists, requires prior specific permission and/or a fee. Request permissions from Permissions@acm.org.

CVMP'14 13-14 November 2014, London, United Kingdom landscapes is available in abundance on the internet. Hence, in this work we propose a practical approach for rerendering of available landscape photographs, such that they can be easily used for modeling natural scenery in realistic rendering applications.

Specifically, we propose a method for estimating view dependent scene depth as well as scene reflectance (albedo) (Fig. 1) from single input landscape images. In this work, we restrict the input to regular low dynamic range (LDR) imagery of landscapes which is commonly found. We estimate scene depth for images with some amount of haze (common case) by examining the global pixel color distribution over the image, particularly the blue channel distribution. For scenes with no haze due to extrmely clear conditions, instead we estimate depth by examining local image color contrast. We combine these estimates with huristics about scanline depth ordering as a way of depth regularization. We demonstrate applications of depth estimation in novel viewpoint renderings, dehazing and digital refocusing. We also demonstrate relighting of landscape imgery, for which we estimate diffuse reflectance of landscapes by estimating the irradiance due the sky in the input photograph. Finally, we also take into account specular reflections on water surfaces which are common in landscape photography and using a semisutomatic process demonstrate relighting of scenes with still water.

To summarize, our contributions for practical landscape rerendering are:

- a simple approach for depth estimation based on blue channel prior for photographs with some haze.

- an approach for depth estimation based on local contrast analysis for photographs with no haze.

- relighting of landscape photographs assuming diffuse reflectance of land and perfect specular reflectance of still water.

\section{RELATED WORK}

While there is significant literature on image based modeling and rendering techniques, we will restrict our discussion here to the most relevant previous work.

Single View Depth Estimation There are a plethora of approaches for estimating scene depth from photographs captured from two or more viewpoints using stereo or structure-from-motion (SFM) techniques. However, scene depth estimation from data captured from a single viewpoint is a much more challenging problem under general conditions. Researchers have employed computational photography techniques such as polarization imaging [12], or coded 


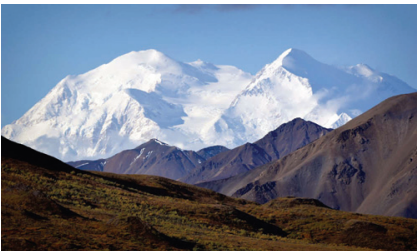

(a) input

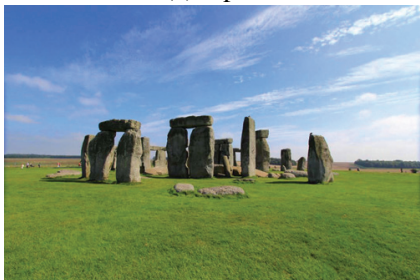

(d) input

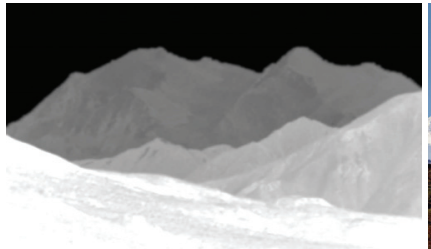

(b) depth map

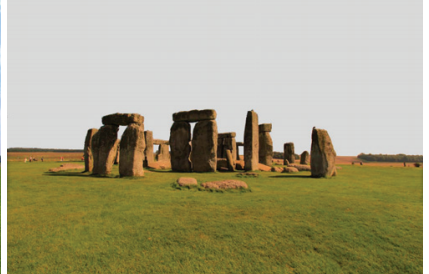

(e) albedo

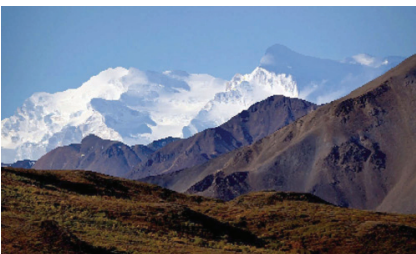

(c) novel view

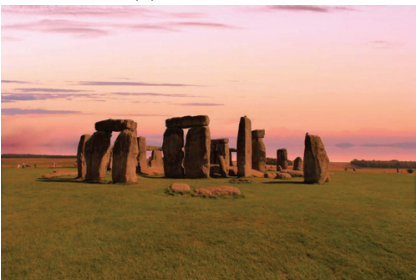

(f) relit

Figure 1: Rerenderings based on estimated depth (a-c) and albedo (d-f).

aperture photography [10] to recover scene depth estimates from single view imagery. However, most relevant to our work is the work of several researchers on the problem of single image dehazing based on various statistical and color channel priors [14, 3, 8, 5]. For images polluted with atmospheric haze, these approaches recover the scene trasmission or depth as part of the dehazing process. Our proposed approach for depth estimation for landscape images is inspired by these approaches. However, we propose a much simpler depth estimation algorithm based on intuitive observations of global color distribution in landscape images. Furthermore, we also propose an approach for depth estimation in landscape images with little or no haze based on local contrast analysis. Researchers have also explored learning based approaches to estimation of depth from single images [11]. Such approaches however require the existence of training data with ground truth depth maps and even then estimate coarse depth maps for test images. Our approach does not require any training data and estimates high resolution depth estimates for given landscape images.

Image-Based Relighting Relighting of scenes typically requires modeling of scene geometry and reflectance, as well as incident illumination. Devebec [1] showed that the incident illumination can be very efficiently and accurately modeled by photographing a mirror sphere placed at the scene to record a light probe, ideally with high dynamic range (HDR) imaging [2]. Such an image based representation of illumination can then be employed in a rendering pipeline to create very realistic rendering results including compositing virtual objects in photographs. In our work, we do not make the assumption of availability of a light probe of the landscape scene since our input is a single photograph of the scene. Hence, we propose to estimate the incident illumination from the photograph itself under the assumption of diffuse reflectance of landscapes. Karsch et al. [6] have proposed a semi-automatic system for estimating incident illumination and reflectance in legacy photographs for realistic insertion of virtual objects. Closely related to our work is that of Lalonde et al. [9], who propose an approach for automatic estimation of directional incident illumination in photographs of outdoor scenes based on a combination of weak cues such as vertical surfaces and shadows, and a data driven prior built from a very large set of internet photographs. Xing et al. [15] have also recently proposed a system for automatic estimation of the environmental illumination in outdoor photographs based on spherical harmonics analysis to recover a low dimensional direc- tional illumination model. These approaches model much more complicated lighting and scene reflectance in photographs using very sophisticated techniques. Instead, we propose a very simple automatic approach for relighting and demonstrate it to work well for natural landscape photographs. Our approach is inspired by the work of Khan et al. [7] who approximate the incident illumination in an image from the visible background. However, a system such as that of Lalonde et al. or Xing et al. could be used to model more sophisticated directional lighting effects in the rerenderings.

\section{ESTIMATING SCENE DEPTH}

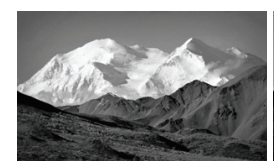

(a) red channel

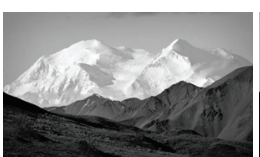

(b) green channel

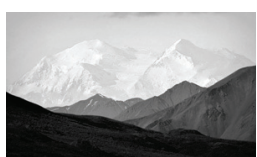

(c) blue channel
Figure 2: Separation of shading and transmission by wavelength.

We propose a simple approach for depth estimation in landscape photographs inspired by recent work in single image dehazing [3, 5]. Most landscape images have a little amount of haze in them due to atmospheric scattering. Fattal [3] has proposed an approach for dehazing based on an optimization that enforces that the surface shading and transmission functions are locally statistically uncorrelated. We make the observation that in typical photographs of natural landscapes, the surface shading and transmission are inherently separated by wavelength into the red/green and blue channels of the image respectively. This can be seen in Fig. 2 for the mountain landscape in Fig. 1, where the shading information is dominant in the red channel while the blue channel contains almost no shading information. This is because most natural landscapes reflect light in the red and green channels but not in the blue channel. The blue channel in these images is mostly dominated by atmospheric airlight and the background sky illumination. This results in the blue channel of the images to progressively increase in magnitude with depth due to haze accumulation. Note that this is true even for scenes without significant amount of haze as seen in the mountain example in Fig. 1. We call this the blue channel prior for landscape images. Given that haze accumulation is typically exponential with depth, we estimate depth as $1-\log (B)$ where $B$ is the blue channel contribution in $[0,1]$ (Fig. $2, \mathrm{c})$. We apply some post-processing to the obtained depth map as a way of regularization including bilateral filtering and depth interoplation across scanlines to handle out- 


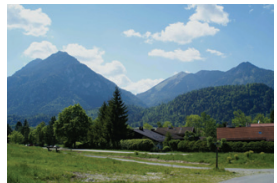

(a) input

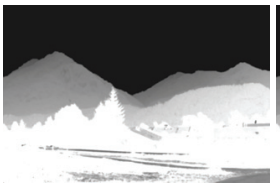

(b) initial depth

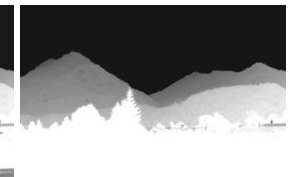

(c) processed depth
Figure 3: Post-processing of initial depth values.

liers. These outliers are typically foreground objects such as rocks or stones, or man made objects such as roads (Fig. 3, b). Hence, we employ additional heuristics about scanline depth ordering to filter the initial depth values. This is based on the observation than in typical landscape photography, the horizon is higher in the frame and forground objects are lower in the frame. This imposes a soft constraint in increasing depth ordering from the bottom to the top scanlines in the image.

He et al. [5] have recently proposed a simple and effective approach for dehazing based on the dark channel prior. The idea is that in image pixels with haze, the difference between the bright color channel and the dark color channel is reduced due to airlight and this color channel difference is a cue for depth estimation. However, we believe our proposed blue channel prior is an even simpler approach for depth estimation in landscape images and demonstrate it to work better than the dark channel prior in scenes with low concentrations of haze (Section 5).

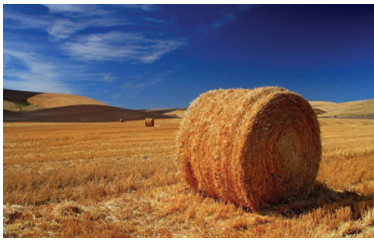

(a) input

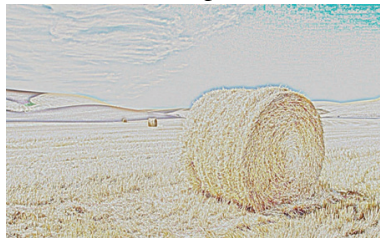

(c) high freq.

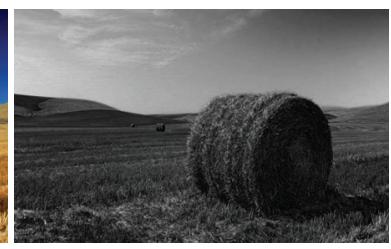

(b) blue channel

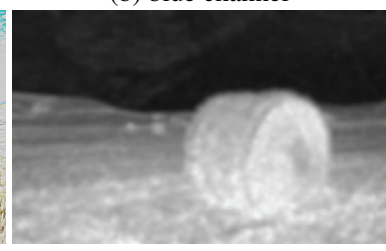

(d) depth map
Figure 4: Depth estimation in scene with no haze.

Some scenes have little or no haze due to very clear atmospheric conditions or due to relatively low depth variation in the scene for any significant haze accumulation. For images of such scenes with negligible haze, the blue channel may not be a very good cue of scene depth. This can be seen in Fig. 4, b. For such scenes, we propose to estimate depth as inversely proportional to the local contrast around every pixel. Local image contrast has been exploited as a depth cue previously for single image dehazing [14]. Instead, we apply local image contrast analysis for depth estimation in clear landscape images. The idea behind this is that even in scenes with no haze, the local contrast around pixels is typically reduced with depth due to foreshortening and camera focus. We employ a variant of relative contrast analysis by first dividing the original image by a blurred (Gaussian kernel) version of the image to obtain an image with accentuated high frequencies (Fig. 4, c). The insight here is that a constant blur kernel over the image still acts as a spatially varying depth dependent blur over the scene, thus impacting the obtained high frequencies based on scene depth. An advantage

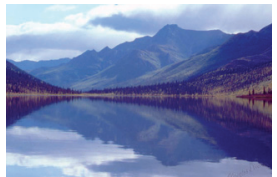

(a) input

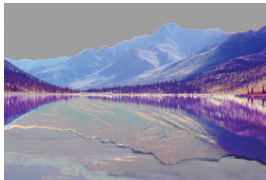

(b) albedo

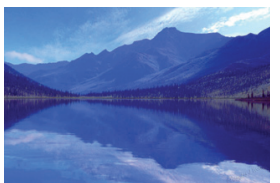

(c) relit
Figure 5: Relighting of landscape with still water.

of the division process is that it also normalizes shadow regions in the image with the well lit areas, thereby removing shading effects from the depth computation. We compute an RMS contrast over a local $7 \times 7$ window centered around every pixel in this high frequency image to obtain the scene depth estimate. Finally, we apply a similar post-processing to regularize the obtained depth map for rendering purposes.

\section{RELIGHTING}

For relighting of landscape images, we make the assumption of diffuse reflectance of the scence and upwards facing surface normal. Under this assumption, the recorded photograph is a product of an unknown scene albedo $\rho_{d}$ and the incident irradiance $E$ due to the sky. Hence, we first estimate the sky irradiance $E$. This requires segmenting out the sky which can be done either manually using standard image editing tools or even automatically using scene depth estimates (Section 3). We resample the segmented sky into a latitude-longitude format and then compute the following integral over the upper hemisphere $E=\int_{\Omega} L_{i}\left(\omega_{i}\right) \cos \theta_{i} d \omega_{i}$, to compute the sky irradiance. Once $E$ has been estimated, we divide the input image pixels by the per channel $E$ to obtain the per channel albedo $\rho_{d}$ (Fig. 1, e). Finally, we relight the scene under a different illumination by multiplying $\rho_{d}$ with the incident irradiance due to a novel illumination environment (Fig. 1, f).

Not all landscape scenes are diffuse and scenes with water bodies exhibit specular reflections. We handle still water bodies in this work using a semiautomatic procedure. First, we assume a horizontal axis of reflection in the image which is typical for most landscape images (Fig 5, a). This reflection axis in a given image is determined based on user input. If we have access to camera parameters, one approach may be to trace rays into the scene to estimate the reflection in the water. Snavely et al. [13] have shown that its possible to compute camera parameters from single input images. This approach would however be very sensitive to the accuracy of the estimated camera parameters for the purpose of relighting and susceptible to calibration errors which can lead to artifacts in the relit water surface. Hence, we take a simpler approach of assuming near orthographic camera view and perfect reflection of the land/sky pixels above the horizontal reflection axis. We also manually segment the water pixels in the photograph from the land/sky pixels for appropriate relighting. Given that the water's specular albedo modulates the reflection, we need to estimate the water albedo next. We follow two separate approaches for relighting water surfaces: For surfaces with slight ripples, we compute a per pixel specular albedo by dividing water pixels by corresponding land/sky pixels above the reflection axis. Next, we relight the diffuse land pixels as before and composite the novel sky. Finally, we relight the water pixels by multiplying the estimated water albedo with the relit reflected land/sky pixels (Fig. 5). However, for perfectly still water surfaces, the above approach may be prone to artifacts due to misalignment between water reflection and land/sky pixels in the absence of an accurate camera model. Hence for scenes with perfectly still water, we instead compute the 


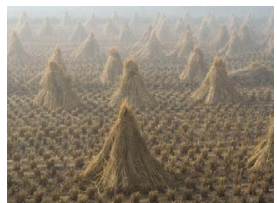

(a) input

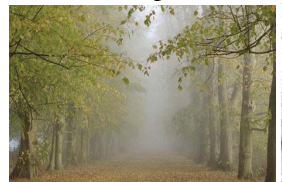

(a) input

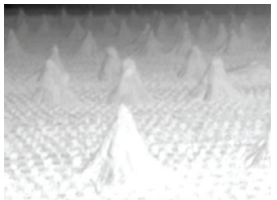

(b) blue channel prior

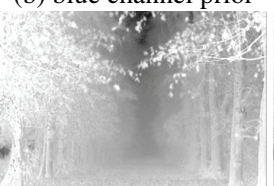

(b) blue channel prior

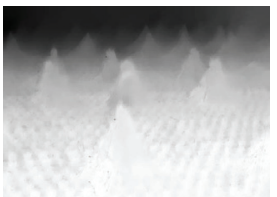

(c) Fattal [3]

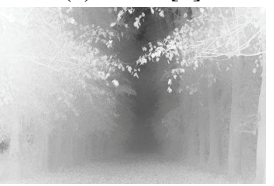

(c) He et al. [5]

Figure 6: Depth estimation with blue channel prior compared to dehazing algorithms.

average reflectivity of the water surface compared to the land/sky above the axis of reflection and employ this average water albedo for relighting the clearl water surface as before (Fig. 10, bottomrow). Our approach produces very plausible results for still water reflections without requiring explicit camera parameters or inverse ray tracing.

\section{RESULTS}

We now present some results of our approach for rerendering landscape photographs as well as some analysis in this section. We use standard LDR images of landscapes downloaded from the web as input. Fig. 1 presents an example of depth estimation with our proposed blue channel prior for a mountain landscape, and demonstrates a rerendering from a novel viewpoint based on the estimated depth map. We also present an example of relighting of a diffuse landscape scene (Stonehenge) in Fig. 1, where the blue sky in the input photograph is used to estimate the albedo of the scene which is then used for relighting the scene under a crimson sky at dusk.

Fig. 6 presents comparison of depth estimation using our proposed blue channel prior with that produced using recent state of the art dehazing algorithms of Fattal [3] and $\mathrm{He}$ et al. [5]. As can be see, our approach produces very comparable depth maps for such scenes. The only noticeable difference is that the blue channel prior results contain a slight amount of residual shading information compared to the results of the dehazing techniques. However, a big advantage of our approach is that depth values are estimated with a much simpler algorithm that is easily implementable.

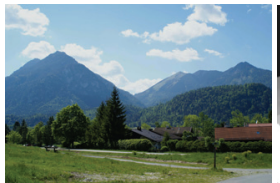

(a) input

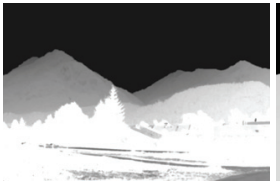

(b) blue channel prior (c) dark channel prior

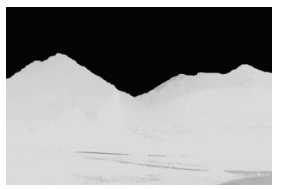

Figure 7: Depth estimation for scene with light haze.

Fig. 7 presents comparison of depth estimation for a scene with light haze using our proposed blue channel prior with that produced using the dark channel prior of $\mathrm{He}$ et al. [5]. Here, we show the initial result without any depth post-processing. As can be see, our approach produces a much better result for this situation than the dark channel prior which has been shown to work well for scenes with dense haze.

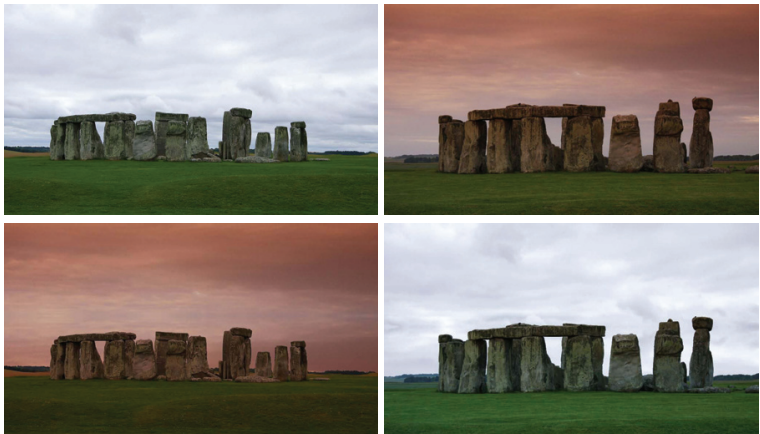

Figure 8: Relighting cross-validation. Top-row: original images. Bottom-row: relit images.

Fig. 8 presents evluation of our proposed relighting strategy for landscape photographs through cross validation. The top-row shows two original photographs of Stonehenge at different times of day. The bottom-row presents relit results where we have relit the landscape of one image with the sky of the other image and vice versa. As can be seen, even when using LDR photographs to estimate sky irradiance, we obtain very plausible relighting results with our approach.

Fig. 10 presents additional results of rerendering of landscape photographs using estimated depth based on the proposed blue channel prior (first three rows) and local contrast analysis (fourth row). For the refocusing example in the second row, we increasingly blurred the pixels in the original image based on the estimated depth map. It should be noted that in scenes with small amount of haze, the blue channel values directly correspond to haze density. This allows us to dehaze the original image simply by subtracting a portion of the blue channel value from all three color channels of the original image (Fig. 10, g-i). Fig. 10 also present additional results for relighting of landscape photographs based on estimated diffuse albedo for land and specular albedo for still water (bottom two rows respectively). As can be seen, we achieve very realistic rerendering results for a variety of landscape scenes using our proposed approach which also has the advantage of being very practical and simple to implement.

\subsection{Limitations}

Our approach certainly has some limitations. Our blue channel prior for depth estimation is based on the observation that most natural scenes do not reflect much light in the blue spectrum other than due to haze accumulation or in the background sky. However, scenes with man made objects like buildings and roads, or bright white objects in the foreground such as rocks or stones tend to violate the blue channel prior's assumptions (Fig. 3 b). In many cases, our proposed post-processing step can help fix errors in depth estimates due to these outliers. However, there can be some difficult cases that are not easily resolved in these situations. Hard shadows in depth discontinuities due to a dominant lightsource such as the sun can also give rise to bias in the depth estimates when using the blue channel prior. However, the approach is still very successfull in providing a consistent depth estimate for a complex scene with little atmospheric haze as shown in Fig. 9.

Our relighting step assumes a diffuse flat world lit by low frequency sky illumination. For scenes with lots of hight variation and highly directional illumination such as the setting sun incident on only one side of a mountain, our simple relighting approach may not 

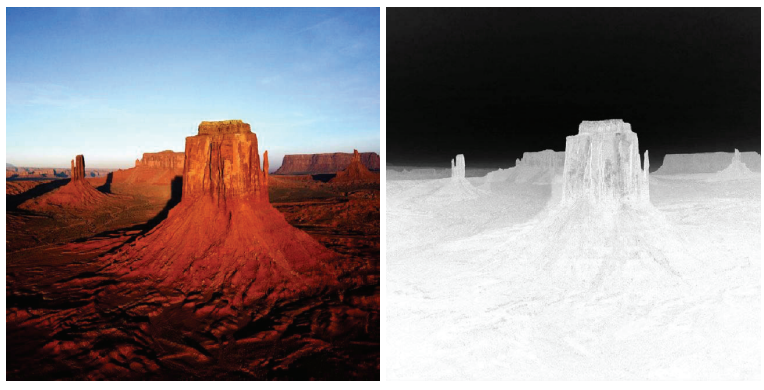

(a) image

(b) depth from blue channel

Figure 9: Hard shadows in depth discontinuities can cause bais in depth estimate.

produce realistic results. Highly directional incident illumination in the original photograph can also result in harsh shadows which are currently baked into the relighting result (Fig. 1 bottom-row). More sophisticated relighting systems (e.g., [6], [15]) can be employed in such cases to obtain better results in such cases. Our approach for relighting still water surfaces makes the assumption of near orthographic camera perspective in the input photograph. For photographs with a more significant wide perspective, our simple approach of reflection about a horizontal axis may not be sufficient to obtain the correct reflection result. Accurate camera parameters may need to be estimated using an approach such as that of Snavely et al. [13] for an inverse ray tracing approach for relighting such photographs. Finally, we currently assume near perfect specular reflection on water and do not model rough specular reflections which are also common on water surfaces.

\section{CONCLUSION}

We have proposed a practical approach for rerendering landscape photographs in this work. Central to our approach is depth estimation from single input images either based on global intesity distributions in the blue channel for scenes with haze or local contrast analysis for haze-free scenes. We show several applications of depth dependent rerenderings of landscape scenes. We further demonstrate relistic relighting of landscape images assuming diffuse reflectance of the scene based on estimating the sky irradiance from the input photograph. We also model perfect specular reflection on still water surfaces and relight scenes with water using a semiautomatic process. We achieve high quality rerendering results using very simple techniques that are easy to implement and hence could easily be incorporated in a post-production pipeline for image-based modeling and rendering of natural landscapes. The proposed techniques could also be easily incorporated as a plugin into image editing softwares. In future work, it would be interesting to combine our relighting approach under natural illumination with additional observations under flash photography similar to the approach Glencross et al. [4] in order to estimate any non-Lambertian surface reflectance for relighting natural scenes.

\section{ACKNOWLEDGEMENT}

We thank Borom Tunwattanapong, David Elsey and Santa Datta for help and support during the project. This project was supported by a Royal Society Wolfson Research Merit Award.

\section{REFERENCES}

[1] P. Debevec. Rendering synthetic objects into real scenes: Bridging traditional and image-based graphics with global illumination and high dynamic range photography. In Proceedings of ACM SIGGRAPH 98, 1998.

[2] P. E. Debevec and J. Malik. Recovering high dynamic range radiance maps from photographs. In Proceedings of ACM SIGGRAPH 97, pages 369-378, 1997.

[3] R. Fattal. Single image dehazing. ACM Trans. Graph., 27(3):72:1-72:9, Aug. 2008.

[4] M. Glencross, G. J. Ward, F. Melendez, C. Jay, J. Liu, and R. Hubbold. A perceptually validated model for surface depth hallucination. ACM Trans. Graph., 27(3):59:1-59:8, Aug. 2008.

[5] K. He, J. Sun, and X. Tang. Single image haze removal using dark channel prior. In $C V P R$, pages 1-8, 2009.

[6] K. Karsch, V. Hedau, D. Forsyth, and D. Hoiem. Rendering synthetic objects into legacy photographs. ACM Trans. Graph., 30(6):157:1-157:12, Dec. 2011.

[7] E. A. Khan, E. Reinhard, R. W. Fleming, and H. H. Bülthoff. Image-based material editing. ACM Trans. Graph., 25(3):654-663, July 2006.

[8] L. Kratz and K. Nishino. Factorizing scene albedo and depth from a single foggy image. In Proc. International Conference on Computer Vision (ICCV), pages 1701-1708, 2009.

[9] J.-F. Lalonde, A. A. Efros, and S. G. Narasimhan. Estimating the natural illumination conditions from a single outdoor image. International Journal of Computer Vision, 98(2):123-145, 2012.

[10] A. Levin, R. Fergus, F. Durand, and W. T. Freeman. Image and depth from a conventional camera with a coded aperture ACM Trans. Graph., 26(3), July 2007.

[11] A. Saxena, S. H. Chung, and A. Y. Ng. 3D depth reconstruction from a single still image. IJCV, 76(1):53-69, Aug. 2007.

[12] Y. Y. Schechner, S. G. Narasimhan, and S. K. Nayar. Instant dehazing of images using polarization. In $C V P R$, pages 325-332, 2001.

[13] N. Snavely, S. M. Seitz, and R. Szeliski. Photo tourism: exploring photo collections in 3D. ACM Trans. Graph., 25(3):835-846, July 2006.

[14] R. Tan. Visibility in bad weather from a single image. In CVPR, pages 1-8, 2008.

[15] G. Xing, X. Zhou, Q. Peng, Y. Liu, and X. Qin. Lighting simulation of augmented outdoor scene based on a legacy photograph. Computer Graphics Forum (Proc. Pacific Graphics), 32(7), 2013. 


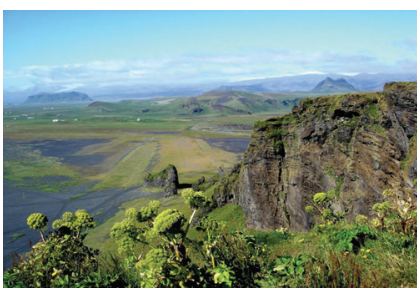

(a) input

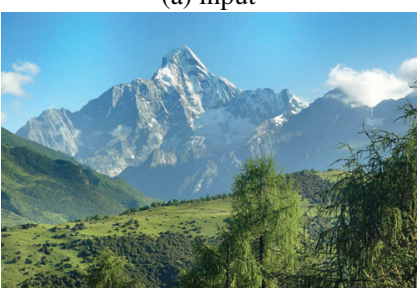

(d) input

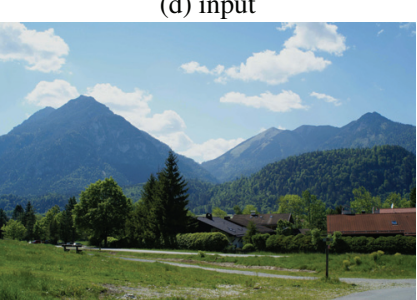

(g) input

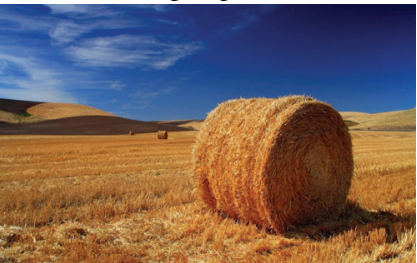

(j) input

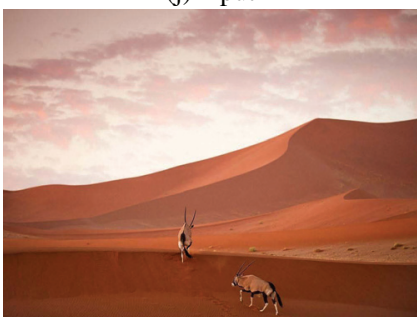

(m) input

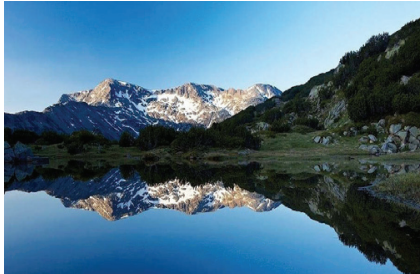

(p) input

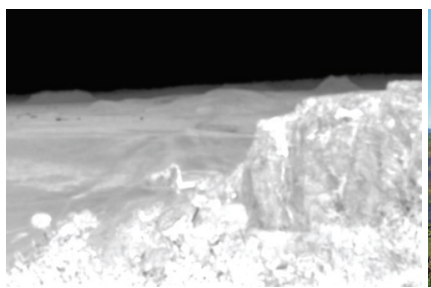

(b) depth map

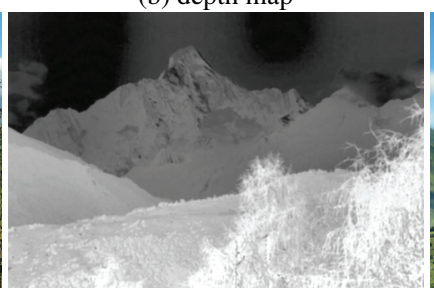

(e) depth map

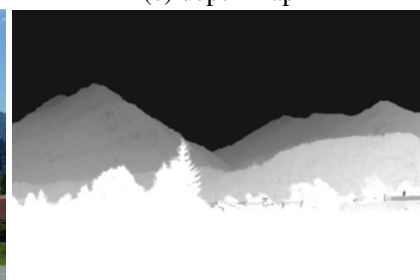

(h) depth map

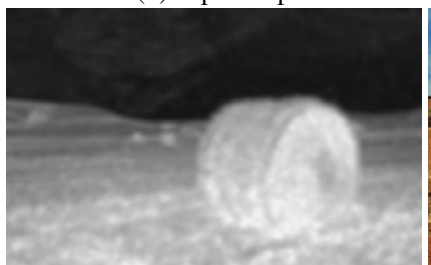

(k) depth map

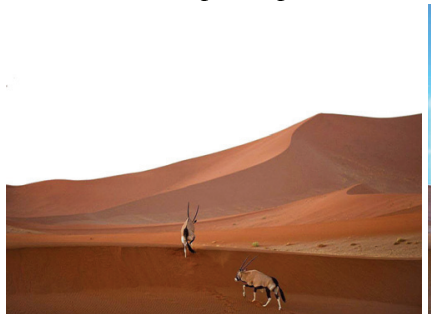

(n) albedo

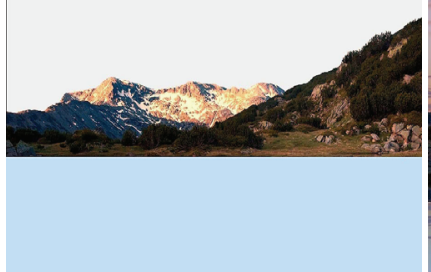

(q) albedo

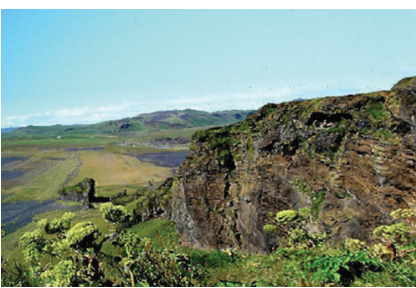

(c) novel view

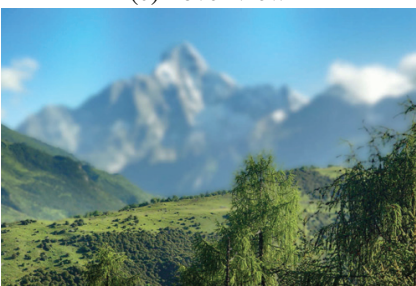

(f) refocused

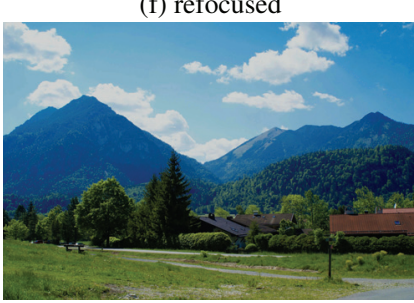

(i) dehazed

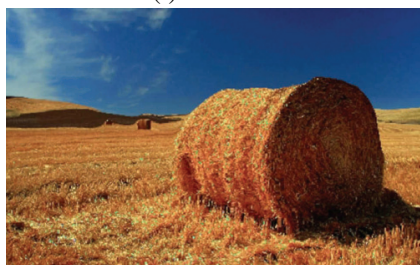

(1) altered perspective

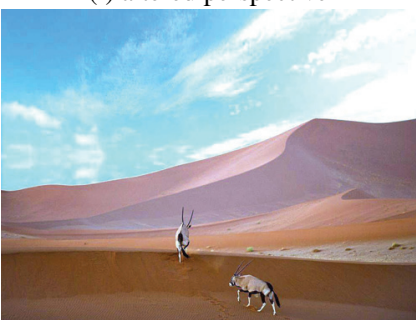

(o) relit

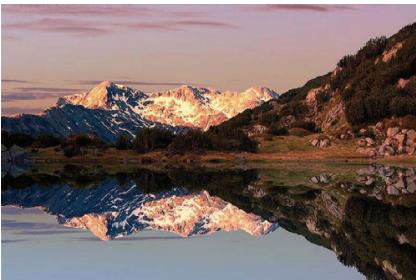

(r) relit

Figure 10: Rerendering examples of landscape photographs. 